EPiC Series in Language and Linguistics
Volume 2, 2017, Pages 14-22
Professional and Academic Discourse:
an Interdisciplinary Perspective

\title{
Textual Enhancement and the Acquisition of the Spanish Present Subjunctive Form
}

\author{
Mariche G. Bayonas \\ University of North Carolina Greensboro, USA. \\ megarcia@uncg.edu
}

\begin{abstract}
Previous empirical research has yielded inconclusive findings on the effectiveness of textual enhancement in drawing learners' attention to target forms and in facilitating their acquisition. The present study focuses on the role of textual enhancement in the recognition, comprehension, and production of Spanish L2 verb forms that differ in their perceptual saliency (communicative value). Spanish students read an enhanced or an unenhanced text containing regular and irregular present indicative and present subjunctive forms. Reading was immediately followed by recognition, comprehension, and production tasks. The resulting data were statistically analyzed in order to ascertain whether they supported or refuted three research hypotheses. Results showed no effect of TE in the three treatments employed.
\end{abstract}

\section{Introduction}

Research on second language acquisition (SLA) has taken many forms in the last decades. One of these forms consists of studies on input and how it is processed, acquired, or manipulated. The importance of input in SLA and the necessity of finding ways to make input more prominent or salient to second language (L2) learners is the focus of many investigators and language instructors (Lightbown, 1991, Sharwood Smith, 1991, 1993, 1994; Spada, 1997). Input is now well established as an essential component in SLA, as a key element if acquisition is to occur. Therefore, understanding the nature of input and how it can be presented to the student in the most effective way is vital in L2 research. When input is made more salient to the learners they are more likely to pay attention to it and thus it can become intake (Schmidt, 1995, 2001; VanPatten, 1996).

Learners acquire the L2 by listening, negotiating meaning in the L2 (oral communication), writing, and reading. The latter permits researchers to control the type of input provided to the learner. Reading, along with listening, is the only other viable way to tender input to language learners. Reading, therefore, allows the researcher to manipulate the input to seek efficient manners of presenting it to learners. One of the methods to manipulate input is by modifying the text itself, in particular by enhancing some grammatical or other forms that may otherwise present a difficulty in learning. This 
modification is now widely known as textual enhancement (TE), which is part of a larger area of research examining input enhancement, whereby input is somehow modified to draw the learners' attention to the form presented.

The current investigation sought to find whether the Spanish subjunctive could be enhanced in such a way that it might make students learn it or intake it more adequately than if presented with no enhancement. In particular, the research questions to be investigated in this paper are the following:

1. Can textual enhancement have an effect on the learning of Spanish present subjunctive?

2. Is the effect of textual enhancement on present subjunctive more robust on irregular forms and regular forms, due to saliency? and saliency:

The research question generates the following predictions concerning items recalled, noticing,

- Hypothesis 1. Textual enhancement has an effect on the number of target items recalled by learners in both English and Spanish among learners that had not been previously exposed to this form.

- Hypothesis 2. Textual enhancement has an effect on "noticing and subsequent recognition" of the Spanish present subjunctive among learners that had not been previously exposed to this form.

- Hypothesis 3. Textual enhancement has a greater effect on salient (irregular verbs) rather than non-salient (regular verbs) forms.

\section{Input Enhancement}

In order to make input more salient to the learners while reading, textual enhancement (TE) has been employed in numerous investigations. Textual enhancement is the technique whereby certain words in reading passages are made more prominent by underlining, bolding, using italics or using different colors, among other possibilities. TE is a type of "input enhancement", also known as visual input enhancement (Izumi 2002, 2003), a pedagogical approach utilized in SLA to draw the learners' attention to particular words. These words are typically ones that have a grammatical function in addition to their own lexical meaning. By drawing attention to forms it is argued that learners can "notice" them better, thus having a positive impact in processing the grammatical form. Researchers have explained that "noticing" or attending to input is the only viable way to have an impact in acquisition (VanPatten, 1996; Gass, 1997; Schmidt, 1994a,b,1995, 2001). The Noticing Hypothesis, put forward by Schmidt (1990, 1995, 2001) illustrates that noticing or awareness is crucial for input to become intake. Furthermore, comprehension precedes production in SLA (Lightbown, 2000) therefore learners need to be exposed to the form prior to even be able to produce it, as is wrongfully assumed by some instructors. TE "provides learners with supplementary doses of comprehensible input and boost the likelihood that they will notice what they need to in order to enhance the process of SLA" (Wong, 2005).

The current study examined the effect of TE and intake of the Spanish present subjunctive, and the effect of TE noticing and recognition of this Spanish verb form among learners that had not previously been exposed to this form. The Spanish subjunctive is a difficult form for learners because it has no direct translation or word in English. The actual subjunctive mood does not add lexical meaning to the sentence that contains it other than doubt, disbelief, etc., under certain contextual circumstances. The Spanish subjunctive, hence, is considered a grammatical form with little communicative value from the standpoint of the principles of processing instruction. Forms that are redundant or do not have lexical meaning per se are considered of "low communicative value” (Van Patten, 96). 


\section{Textual Enhancement and Second Language Research}

The effect of textual enhancement on perception and recall of information has been investigated both with regard to L1 and L2. Some authors found no positive influence of TE on the acquisition or noticing of form (Shook, 1994; Alanen, 1995; Jourdenais et al. 1995, Izumi, 2003; Lee, 2007), whereas others uncovered inconclusive results regarding TE's impact on the acquisition or noticing of L2 forms (Leow, 2001; Leow et al,. 2003; Overstreet, 1998; Wong, 2000, 2003). Languages that have been analyzed are English, French, and Spanish with learners of numerous first language backgrounds (English, Arabic, Korean, Japanese, etc.).

The effect of TE in the acquisition of Spanish has received some focus (i.e. Jourdenais et al., 1995; Leow, 1997; Overstreet, 1998; Shook, 1994; Leow 2001; Leow et al., 2003). Shook (1994) examined the effect of direct enhancement with the present perfect tense and relative pronouns. He designed several tasks to assess the processing of input into intake by first and second-year students of Spanish. His results indicated that students process grammatical items as intake if they have their attention explicitly drawn to them. Additionally, students processed input for meaning before the form.

Jourdenais et al. (1995), like Shook (1994) also investigated the role of TE among preterit and imperfect verb forms in Spanish, and TE was achieved by bolding, shading and underlining the target verb forms. Participants were assigned to enhanced or unenhanced groups and were subsequently asked to write picture-based narrative similar to the one previously read. During this last task, think aloud protocols were collected. The data analyzed revealed that the think-aloud protocols of the enhanced group contained more episodes related to the verbs than those of the unenhanced group. In addition the former also produced more target features in their written production. Thus, their results indicated that TE promoted noticing, which has an effect on subsequent output. Leow, (1997) on the other hand, did not reach conclusions that echoed the positive outcomes of Jourdenais et al. (1995) and Shook (1994). He investigated the effect of input enhancement and text length on comprehension and intake in SLA. Participants were exposed to four conditions: long, short, enhanced, and non-enhanced versions of the same text. His results revealed no significant effect of input enhancement on comprehension or intake. He claimed that his results raise questions as to the effectiveness of external manipulation of written input in the facilitation of learners' comprehension and processing of linguistic forms. A negative effect of TE was also reported by Overstreet (1998). In his study he analyzed TE and content familiarity so participants were exposed to four text conditions: familiarity, non-familiarity, enhancement or unenhancement. Not only did he not find a positive effect of TE but in fact these conditions were found to affect the learners' comprehension negatively. He concluded that TE took the attention away from the comprehension resulting in more attention to form than meaning. A subsequent study by Leow et al. (2003) focused on TE and type of linguistic item as it related to comprehension and intake. Leow et al. also employed think-aloud protocols, and they investigated the Spanish present perfect and Spanish present subjunctive forms. Recognition and comprehension tasks that followed the treatment were quantitatively analyzed, and resulted in no significant benefits of TE for noticing, comprehension, and intake. However, they did find significant benefits on salient (present perfect) over less salient (present subjunctive) linguistic forms.

Wong (2003) conducted a study where French was the L2 language under investigation. In particular, she investigated how TE, more specifically increasing the comprehensibility of input via simplified input (SI) affected the acquisition of the French past participle agreement in relative clauses. She exposed participants to four treatments combining TE and SI and her results led her to conclude that TE was not effective with regard to the acquisition of the French form. Unlike the results obtained by Leow et al., whereby TE did not affect comprehension, Wong found that TE resulted in better recall of enhanced information. 
In light of the previous findings the current research was undertaken to further test the effect and validity of TE on students' comprehension, noticing and production of a grammatical form: the Spanish present subjunctive.

\section{Methodology}

\subsection{Participants}

The total number of participants in this study was 86 undergraduate students in their first semester of Spanish at the University of North Carolina Greensboro. Participants were English native-speakers and they were studying Spanish as a second language. All participants were administered a grammar test to measure their Spanish ability. Only the data from participants who scored 10/20 or below in the grammar test were included in the study while the data from the participants who scored $11 / 20$ or above $(n=11)$ were eliminated in order to exclude students who might have been exposed to the subjunctive form prior to taking this class. Participants had to complete all tasks of the experiment in order to be included in the final pool. Six participants did not respond to some of the tasks and their data were not incorporated, which left the final number of participants to 69.

\subsection{Verb Forms}

The forms under study were Spanish present subjunctive, both in the regular (less salient) and the irregular (more salient) form. Spanish subjunctive is considered a form with low communicative value because its meaning cannot be translated into English, and it does not add any lexical meaning to the actual content of the verb. It is used only under very specific circumstances, for example to express doubt, wish, etc. For example, in order to express the following sentence: "The professor wants the students to finish their homework", one has to use the subjunctive form for the verb "to finish". The subjunctive form has, thus, no inherent semantic value and it can be classified as having low communicative value.

\subsection{Procedure}

Data were collected during students' regular Spanish class. The researcher explained the procedure and asked for volunteers to stay. Those students who stayed were distributed the reading texts randomly (the investigator distributed one enhanced and one un-enhanced form sequentially) and allotted all the time the participants needed to read the text. Upon finishing, they raised their hand and the investigator picked up the reading text and gave them a set of 4 tasks to complete: grammar, task 1,2 , and 3 . Once they finished, they handed the package back and left the classroom at their leisure. There were no time constrains but all students took forty-five minutes or less to complete all tasks. 


\subsection{Materials}

\subsubsection{Reading Text}

The text selected for this study was presented in two treatments: enhanced (E) or unenhanced (U). The E text had all subjunctive verb forms enhanced with CAPITAL LETTERS and BOLD. There were 10 instances each of the regular present subjunctive and irregular present subjunctive. The $U$ text contained the same information but no verbs were textually enhanced. In total there were ten instances each of present subjunctive regular, present indicative regular, present subjunctive irregular, and present indicative irregular.

\subsubsection{Grammar Text}

Students were first asked to complete a grammar test. This test contained 20 questions that varied in level of difficulty. Students that scored ten points or below were placed in the "beginner" group. Students that scored eleven points or above were placed in the "intermediate” group.

\subsubsection{Task 1}

The first task consisted of a free written recall in English. Participants were asked to write as many ideas as possible from the text they had just read. These data were analyzed for overall number of English (translated) grammatical tokens recalled. This task was performed in the students' first language.

\subsubsection{Task 2}

Task 2 was a free written recall in Spanish. It immediately followed task 1, and it asked participants to write down in Spanish as many ideas as they remembered from the text they had read. These data were analyzed for overall number of Spanish grammatical tokens recalled.

\subsubsection{Task 3}

This task was a recognition task. It contained a passage not related to the text read with 20 verbs in both the indicative and subjunctive forms where students had to circle the right verb form according to the context. These data were analyzed for overall correctness.

\section{Analyses}

The data obtained from all tasks were computed individually and submitted to independent sample t-tests for equality of means and they indicated the following results. For all tasks the only data analyzed were the tasks completed by students who were in SPA 101 and did not pass the grammar test. The total number of participants whose score was tallied was 69, of whom 34 received a U version of the reading text and 35 received an E version of the reading text. The tables thus indicate results by tasks. Tables also display whether students read a text with TE or No TE. They, likewise, illustrate the results with regard to SR (Subjunctive Regular verbs) or SI (Subjunctive Irregular verbs).

Task 1 was a free written recall in English, and every occurrence of a verb that was in the Subjunctive in the reading text was tallied. Both regular and irregular verb forms were analyzed. Table 1 illustrates the results obtained from the independent-samples t-tests, and there were no significant differences between the $\mathrm{E}$ and $\mathrm{U}$ groups for both irregular and regular subjunctive verbs. In other words, participants exposed to the E text did not significantly recall more irregular or regular verb forms than participants who were exposed to the U text.

Task 2 was also a written recall but in Spanish instead of English. The results displayed in Table 1 from the independent t-tests showed no significant values for subjunctive regular verbs but they show significance for the subjunctive irregular verbs. Similar to the results obtained for task 1 , there were no 
significant differences between the $\mathrm{E}$ and $\mathrm{U}$ groups among regular and irregular subjunctive verb forms, which indicated that TE did not affect performance in task 2.

\begin{tabular}{cccccc}
\hline Variable & $\begin{array}{c}\text { Unenhanced } n=34 \\
\mathrm{M}(S D)\end{array}$ & $\begin{array}{c}\text { Enhanced } n=35 \\
\mathrm{M}(S D)\end{array}$ & $t$ & $d f$ & $p$ \\
\hline T1SI & $.32(.59)$ & $.51(.78)$ & -1.143 & 67 & .02 \\
T1SR & $.65(.73)$ & $.57(.82)$ & .405 & 67 & .67 \\
T2SI & $.09(.38)$ & $.26(.51)$ & -1.567 & 67 & $.00^{*}$ \\
T2SR & $.24(.43)$ & $.24(.43)$ & .000 & 66 & 1.00 \\
\hline
\end{tabular}

Table 1. Independent t-test for post-test scores on recall for Subjunctive Irregular and Regular Verbs Note. Equal variance assumed. ${ }^{*} \mathrm{p}<.01$

The results for Task 3 are displayed in Table 2. The results are shown for both treatment (TE or no TE) applied to subjunctive and indicative verbs as well as for regular and irregular ones. The table thus illustrates results for Ind Reg (indicative regular verb), Sub Reg (subjunctive regular verb), Ind Irr (indicative irregular verb), and Sub Irr (subjunctive irregular). Table 2 indicates a similar trend among participants exposed to $\mathrm{E}$ and $\mathrm{U}$ texts. Significance is not reached among any of the Spanish verb forms indicating a lack of a difference due to TE on texts. Similar to task 2, however, significance was reached for Task 3 with subjunctive irregular verbs.

\begin{tabular}{lccccc}
\hline Variable & $\begin{array}{c}\text { Unenhanced } n=34 \\
\mathrm{M}(S D)\end{array}$ & $\begin{array}{c}\text { Enhanced } n=35 \\
\mathrm{M}(S D)\end{array}$ & $t$ & $d f$ & $p$ \\
\hline T3 IndReg & $67.28(20.87)$ & $71.07(17.36)$ & -.821 & 67 & .19 \\
T3 SubReg & $36.76(25.55)$ & $34.29(26.49)$ & .40 & 67 & .53 \\
T3 IndIrr & $91.18(19.35)$ & $85.74(25.8)$ & .99 & 67 & .04 \\
T3 SubIrr & $17.65(10.71)$ & $24.29(19.40)$ & -1.75 & 67 & $.00^{*}$ \\
\hline
\end{tabular}

Table 2. Independent t-test for post-test scores on recall for Indicative Regular and Subjunctive Regular Verbs

Note. Equal variance assumed. ${ }^{*} \mathrm{p}<.01$

\section{Discussion}

The first hypothesis was; Textual enhancement has an effect on the number of target items recalled by learners in both English and Spanish among learners that had not been previously exposed to this form. The prediction was not accurate. Participants exposed to TE did not recall more target verbs in either English or Spanish than participants with no TE. Significance was not reached for either regular or irregular verbs indicating that saliency (regular vs. irregular) did not play a role in this part of the study.

For hypothesis number two: textual enhancement has an effect on "noticing and subsequent recognition" of the Spanish present subjunctive among learners that had not been previously exposed to this form, the answer is no. Participants who read the TE text did not performed significantly better in the noticing or recognition task than participants who read the one without TE. In other words, TE did not have an effect on noticing or recognizing the Spanish subjunctive in either regular or irregular form. In this case, saliency did not play a significant role either. 
Finally, the answer to the third hypothesis: textual enhancement has a greater effect on salient (irregular verbs) rather than non-salient (regular verbs) forms, is yes. TE has an effect on irregular verbs on two tasks. In both cases, significance was reached among subjunctive irregular verbs only. Students in this study processed the forms influenced by factors such as saliency, TE, and type of treatment. Both groups exposed to Spanish subjunctive performed similarly at a level of learning in which they had not been previously exposed to this verb form.

The speculation that Overstreet (1998) drew from his results is also present in this study. He suggested that the forms used (Spanish preterit and imperfect) were too salient to actually process them for meaning. However, TE in this study did not have a negative effect on the acquisition of the Spanish verb forms, unlike previous studies. TE did not have any impact whatsoever in noticing, and producing the Spanish subjunctive verb form. But it did have an impact on comprehension of subjunctive irregular forms.

\section{Conclusion}

The following conclusions were reached in light of the results obtained from the current investigation. TE has no effect in the acquisition of the Spanish subjunctive regular verb. However, TE may have an impact on the acquisition of subjunctive irregular verb forms. Participants exposed to a TE text recalled similar number of irregular and regular verb forms in English and Spanish as participants not exposed to a TE text. TE has an effect on low communicative/high saliency items but it does not have effect on low communicative/low saliency items. Saliency, thus, seems to play a role in aiding to acquire Spanish Subjunctive. TE does not play a role either. Results obtained from this study indicate that TE has no effect on comprehension, production, and noticing of Spanish subjunctive verbs, both with respect to regular and irregular verbs. Its low communicative value and low saliency does not accelerate or slow the rate at which it is learned. The low communicate high saliency forms seem to be influenced by TE. Irregularity may help students recall or notice forms that are otherwise very low in communicative value. As for the regular forms, they did not undergo the same effect. All advanced language learners of Spanish learn the forms, whether regular or irregular, but it may seem that at early stages of acquisition, saliency (irregular forms) may be "easier" to notice and comprehended than nosaliency (regular forms). Other factors may come into play when acquiring these grammatical forms. It is speculated that motivation may be one of these factors that aids in acquisition. If learners feel the absolute necessity to learn these low communicative value forms -actually disappearing among some bilingual communities where the language in contact does not have the same counterpart in its grammar (Silva-Corvalán, 2001), it is hypothesized that motivation, along with other treatments such as TE may result in significant effect.

\section{Limitations}

Future research should incorporate a higher number of regular and irregular verbs in the reading text. The intent of utilizing the lower number in this study was to find a balanced number of verbs, ten each of present indicative regular, present indicative irregular, present subjunctive regular and present subjunctive irregular, however it would have been more valuable to incorporate a higher number of the subjunctive ones.

Another weakness of this study was the low number of tokens in Task 3. In order to avoid fatiguing the participants it was decided to only have ten selection items. However, the test itself was shorter than anticipated and a future study would benefit from having more items. 
The possible effect of TE on all types of Spanish subjunctive verbs was hindered by the low number of participants. A higher pool may have added more robustness to the study and might have yielded significant results in areas that did not reach it.

\section{References}

Alanen, R. (1995). "Input enhancement and the rule presentation in second language acquisition". Attention and awareness in foreign language acquisition. Ed. R. Schmidt. Honolulu: University of Hawaii, SLTCC. 259-302.

Gass, S. M. (1997). Input and interaction and the second language learner. MahWah, NJ: Erlbaum.

Izumi, S. (2003). "Visual input enhancement as focus on form". Sophia Linguistica 51: 1-30.

Jourdenais, R., Ota, M., Stauffer, S., Boyson, B. \& Doughty, C. (1995). "Does textual enhancement promote noticing? A think-aloud protocol analysis". ". Attention and awareness in foreign language acquisition. Ed. R. Schmidt. Honolulu: University of Hawaii, SLTCC. 183-216.

Leow, R. (1997). "The effects of input enhancement and text length on adult L2 reader's comprehension and intake in second language acquisition”. Applied Language Learning, 8: 151-182.

Leow, R. (2001). "Do learners notice enhanced forms while interacting with the L2?: An online and offline study of the role of written input enhancement in L2 reading”. Hispania, 84: 496-509.

Leow, R., Egi, T., Nuevo, A. M., \& Tsai, Y.-C. (2003). "The roles of textual enhancement and type of linguistic item and adult L2 learner's comprehension and intake”. Applied Language Learning, 13: $1-16$.

Lightbown, P. (1991). "Focus on form: A design feature in language teaching methodology". In de Bot, K., Coste, D., Kramsch, C., and Ginsberg, R. (Eds.), Foreign Language Research in a Crosscultural Perspective. John Benjamins: Amsterdam. 39-52.

Lightbown, P. (2000). "Anniversary article: Classroom SLA research and second language teaching”. Applied Linguistics, 21: 431-462.

Overstreet, M. (1998). "Text enhancement and content familiarity: The focus of learner attention". Spanish Applied Linguistics, 2: 229-258.

Schmidt, R. (1990). "The role of consciousness in L2 learning”. Applied Linguistics, 11: 205-225.

Schmidt, R., (1994a). "Deconstructing consciousness in search of useful definitions for applied linguistics”. AILA Review, 11: 11-26.

Schmidt, R., (1994b). "Implicit learning and the cognitive unconscious”. In: Ellis, N. (Ed.), Implicit and Explicit Learning of Languages. Ed. N. Ellis. Academic Press: London. 165-209

Schmidt, R., ed. (1995). Consciousness and Foreign Language Learning: A Tutorial on the Role of Attention and Awareness in Learning. In Attention and Awareness in Foreign Language Learning. University of Hawaii: Honolulu. 1-63.

Schmidt, R. (2001). “Attention”. Cognition and second language instruction. Ed. P Robinson. Cambridge: Cambridge University Press. 3-32.

Sharwood Smith, M. (1991). "Speaking to many minds: On the relevance of different types of language information for the L2 learner”. Second Language Research, 7: 118-132.

Sharwood Smith, M. (1993). "Input enhancement in instructed SLA: Theoretical bases". Studies in Second Language Acquisition, 15(2):165- 79.

Sharwood Smith, M. (1994). Second Language Learning: Theoretical Foundations. Longman, New York.

Shook, J. D. (1994). "FL/L2 reading, grammatical information, and the input to intake phenomenon”. Applied Language Learning, 5: 57-93.

Silva-Corvalán, C. (2001). Sociolingüística y Pragmática del Español. Washington, D.C.: Georgetown University Press. 
Spada, N. (1997). "Form-focused instruction and second language acquisition: a review of classroom and laboratory research”. Language Teaching 30: 73-87.

VanPatten, B. (1996). Input processing and grammar instruction: Theory and research. Norwood: NJ: Ablex.

Wong, W. (2000). The effects of textual enhancement and simplified input on L2 comprehension and acquisition of non-meaningful grammatical form. Unpublished dissertation, University of Illinois: Urbana-Champaign, IL.

Wong, W. (2003). "Textual enhancement and simplified input: Effects on L2 comprehension and acquisition of non-meaningful grammatical form”. Applied Language Learning, 13: 17-45.

Wong, W. (2005). Input enhancement: From theory and research to the classroom. New York: McGraw-Hill. 